\title{
Emerging Trends in the Etiology and Antimicrobial Susceptibility Pattern of Enteric Pathogens in Rural Coastal India
}

\author{
Mamatha Ballal1 $^{*}$, Suganthi M. Devadas², Rituparna Chakraborty¹, Vignesh Shetty ${ }^{2}$ \\ ${ }^{1}$ Enteric Pathogens Division, Melaka Manipal Medical College, Manipal University, Manipal, India \\ ${ }^{2}$ Enteric Pathogens Lab, Manipal University, Manipal, India \\ Email: "mamatha_98@yahoo.com, suganthimartena@gmail.com, rituparna2K7@gmail.com, \\ vigneshshetty2009@yahoo.co.in
}

Received 21 February 2014; revised 20 March 2014; accepted 3 April 2014

Copyright (C) 2014 by authors and Scientific Research Publishing Inc.

This work is licensed under the Creative Commons Attribution International License (CC BY). http://creativecommons.org/licenses/by/4.0/

(c) $\underset{\mathrm{EY}}{\mathrm{B}}$ Open Access

\begin{abstract}
Introduction: Gastroenteritis is recognized as a serious public health problem in India. It is a syndrome that can be caused by different bacterial, viral and parasitic pathogens. Indiscriminate use of antibacterial agents has resulted in the development of multidrug resistant organisms. A retrospective study was done to analyze the etiological spectrum of diarrhoea and to determine the antimicrobial resistance pattern of bacterial pathogens. Methodology: Fecal specimens from patients with acute or chronic diarrhoea were received prior antibiotic administration and processed for routine microscopy of parasites; culture, antimicrobial susceptibility testing and polymerase chain reaction for bacterial pathogens and latex agglutination for rotavirus. Results: Out of the 6043 stool samples collected during the period of Jan 2005-Dec 2013, $678(11.2 \%)$ enteric pathogens were isolated. The predominant isolates were Diarrheagenic Escherichia coli (DEC) (46.3\%) followed by Salmonella, Shigella, Vibrio cholerae and parasites. Certain unusual pathogens were also isolated. Among age wise distribution, Diarrheagenic Escherichia coli (DEC) mainly Enteroaggregative Escherichia coli (EAEC) was isolated from $<5$ years of age. The majority of other enteric pathogens were isolated from the adult population. The enteric pathogens isolated showed resistance to multiple antibiotics. The resistance pattern observed during this period showed an overall increase in resistance to the antibiotics tested. Conclusion: The present study documents the overall role of etiological agents as causes of diarrhoea and indicates that the indiscriminate use of potent antibiotics can lead to acquisition of resistance to important therapeutic agents.
\end{abstract}

\section{Keywords}

Diarrheogenic Escherichia coli (DEC), Salmonella, Shigella, Vibrio, Multidrug Resistant

\footnotetext{
${ }^{*}$ Corresponding author.
}

How to cite this paper: Ballal, M., et al. (2014) Emerging Trends in the Etiology and Antimicrobial Susceptibility Pattern of Enteric Pathogens in Rural Coastal India. International Journal of Clinical Medicine, 5, 425-432. 


\section{Introduction}

Global, regional and national estimates place diarrhoeal diseases as a major neglected public health problem. In the Southeast Asian region, almost $48 \%$ of the estimated 3.07 million deaths annually are attributed to acute respiratory infections and diarrhoeal diseases with the highest burden in 5 countries: Bangladesh, India, Indonesia, Myanmar and Nepal where these diseases caused 60,000 deaths annually [1]-[3]. Diarrhoea is a syndrome that can be caused by different bacterial, viral and parasitic pathogens. Understanding the prevalence of these etiological agents is necessary as it contributes to its epidemiology and also to the changing trends in causing gastroenteritis. Indiscriminate use of antimicrobial agents has resulted in development of multidrug resistant organisms [4]. Accurate understanding of the cause of diarrhoea in a given setting is an arduous task that requires systematic monitoring of the various pathogens. A retrospective study was done to analyze the etiological spectrum of diarrhoea and to determine the antimicrobial resistance pattern of bacterial pathogens.

\section{Methodology}

Specimen collection: Fecal specimens from patients with acute or chronic diarrhoea were received for microscopy of parasites and culture of bacterial enteric pathogens from both inpatients and out patients at Diagnostic Microbiology Laboratory, from Jan 2005-Dec 2013. Informed consent was obtained from the patients prior to sample collection. Around 6043 stool specimen were collected before antibiotic administration and processed for identification of various pathogens. No transport medium was employed as the fresh specimen was received in the laboratory within 30 minutes and processed immediately without any further delay using standard laboratory techniques.

Specimen processing: Routine stool microscopy of saline and iodine preparations was examined for intestinal parasites. Smears stained with modified acid fast stain from each specimen were examined for coccidian parasites. A battery of culture media-Alkaline Peptone Water, Selenite Feces broth, Blood agar, Mac Conkey agar, Thiosulphate Citrate Bile salt Sucrose agar (TCBS) were used for the isolation of bacterial pathogens. Bacteriological analysis was done by standard laboratory techniques [5] [6]. Salmonella, Shigella and Vibrio cholerae were serotyped by slide agglutination test using specific antisera (Difco, USA and NICED, Kolkata). Salmonella isolates were sent to Central Research Institute-CRI, Kasauli and NICED, Kolkata for serotyping with specific antisera. Escherichia coli isolates obtained from patients suffering from persistent diarrhoea for more than 2 weeks were subjected to Multiplex PCR targeting the specific virulence genes [7]. Clinical isolates of E. coli were specifically subjected to quantitative biofilm assay to screen for the presence of EAEC [8].

Latex slide agglutination test was used for the detection of Rotavirus in the stool samples (Ranbaxy Labs Ltd, New Delhi).

Antimicrobial susceptibility test: The antimicrobial susceptibility test was performed for Diarrheagenic E. coli, Salmonella, Shigella, V. cholerae and Aeromonas by the Kirby Bauer disc diffusion technique using commercially available disc (Hi-media). The antibiotics used were ampicillin (AMP $10 \mu \mathrm{g})$, chloramphenicol (CHL $30 \mu \mathrm{g}$ ), tetracycline (TET $30 \mu \mathrm{g}$ ), cotrimoxazole (SXT $25 \mu \mathrm{g}$ ), ciprofloxacin (CIP $5 \mu \mathrm{g})$, norfloxacin (NX $10 \mu \mathrm{g}$ ), nalidixic acid (NAL $30 \mu \mathrm{g}$ ), amoxicillin-clavulanic acid (AMC $20+10 \mu \mathrm{g}$ ), gentamicin (GEN $10 \mu \mathrm{g}$ ), amikacin (AK $30 \mu \mathrm{g}$ ), ceftriaxone (CRO $30 \mu \mathrm{g}$ ), and ceftazidime (CAZ $30 \mu \mathrm{g}$ ). The current CLSI guidelines [9] were followed for interpretation of the results and E. coli ATCC 25922 was used as the control strain.

Patients' clinic-demographic details which includes age, sex, ward/OPD, presenting clinical history and underlying illness were noted wherever applicable.

\section{Results}

Of 6043 fecal specimens, 678 (11.2\%) stool specimens were positive for enteric pathogens. Out of the 678 pathogens, 422 (62.2\%) were isolated from males and 256 (37.7\%) from females. Table 1 summarizes the various etiology of diarrhoea. Among DEC, Enteroaggregative E. coli (EAEC), Enteropathogenic E. coli (EPEC) and Enterotoxigenic E. coli (ETEC) were isolated.

In this study, out of the 71 Salmonella isolates (Table 1), 54 (76\%) non-typhoidal Salmonella (NTS) were isolated from diarrheal patients, of which, Typhimurium (21\%), Weltevreden (9\%), Hindmarsh (6\%), Denver (3\%) are the common serotypes and 4 strains (12\%) were untypable. The rare serovars isolated were Oslo, Bovismorbificans and Schleissheim (data not shown). 
Table 1. Etiology of enteric pathogens isolated from 2005-2013.

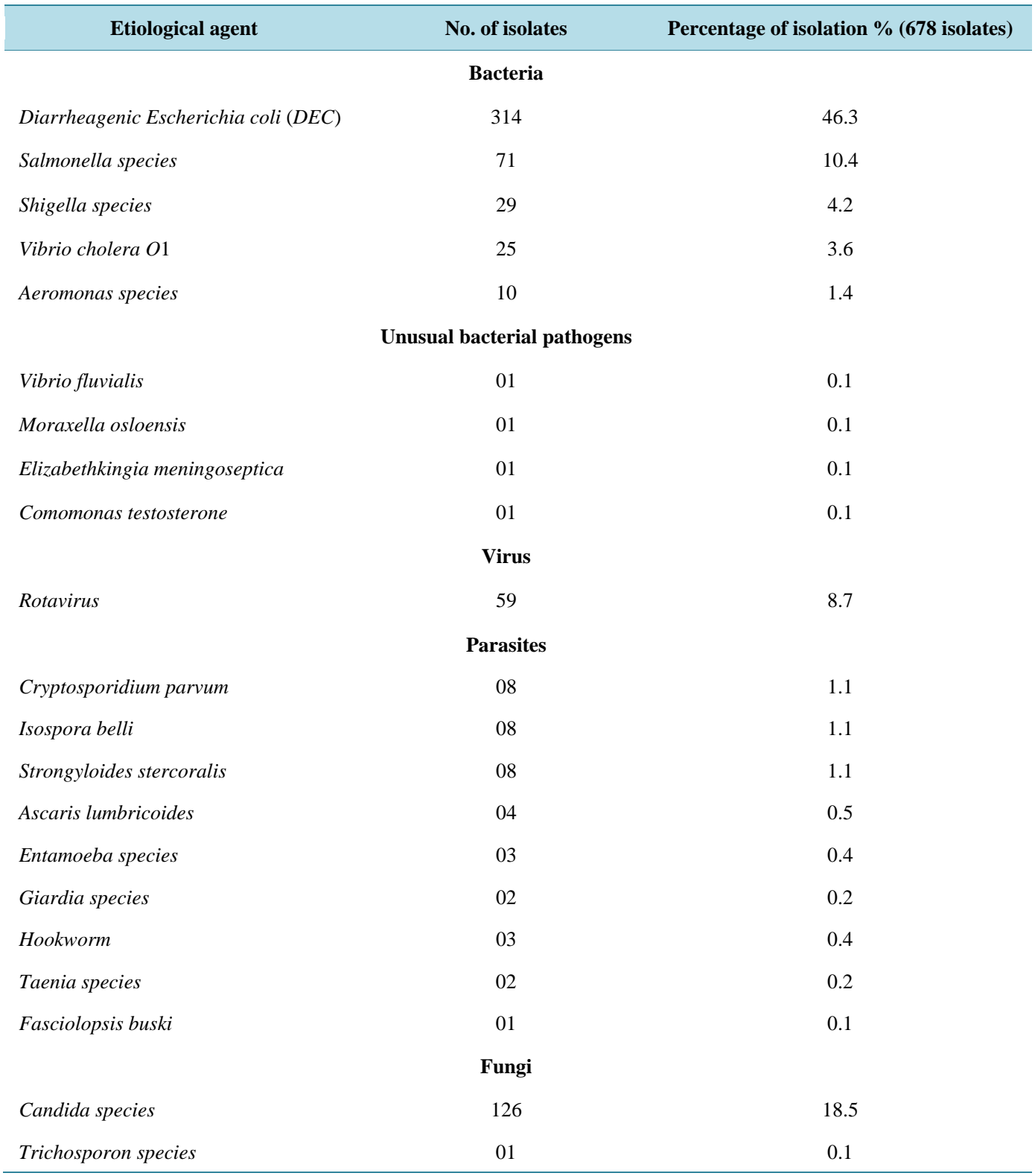

Among Shigella, S. flexneri $2 a$ was the most common isolate (88\%), followed by S. sonnei (10\%), S. dysentriae type $1(1 \%)$ and $S$. boydii (1\%). Out of the $25 \mathrm{~V}$. cholerae isolates, $84 \%$ and $16 \%$ belonged to serotypes Ogawa and Inaba respectively. Aeromonas hydrophila and A. sobriae were among Aeromonas species (Table 1).

The unusual bacterial pathogens, Moraxella osloensis \& Elizabethkingia meningoseptica were isolated from pediatric patients and Vibrio fluvialis and Commomonas testosteroni were isolated from the adult patients. The coccidian parasites (Cryptosporidium \& Isospora) were isolated from HIV infected patients with persistent diarrhea. Fasciolopsis buski was isolated from an HIV infected patient. Candida species (18.5\%) as the sole pathogen was isolated from immunocompromised patients (Table 1).

Age specific distribution of bacterial enteric pathogens: The age wise association of various bacterial enteric pathogens are shown in Figure 1. Among DEC, EAEC was high (40\%) among children $<5$ years of age, whereas EPEC and ETEC were more among the adult group (data not shown). Rotavirus diarrhea (8.7\%) was seen in $<2$ years of age. Parasites and yeast isolates were predominately seen in adult age group. 


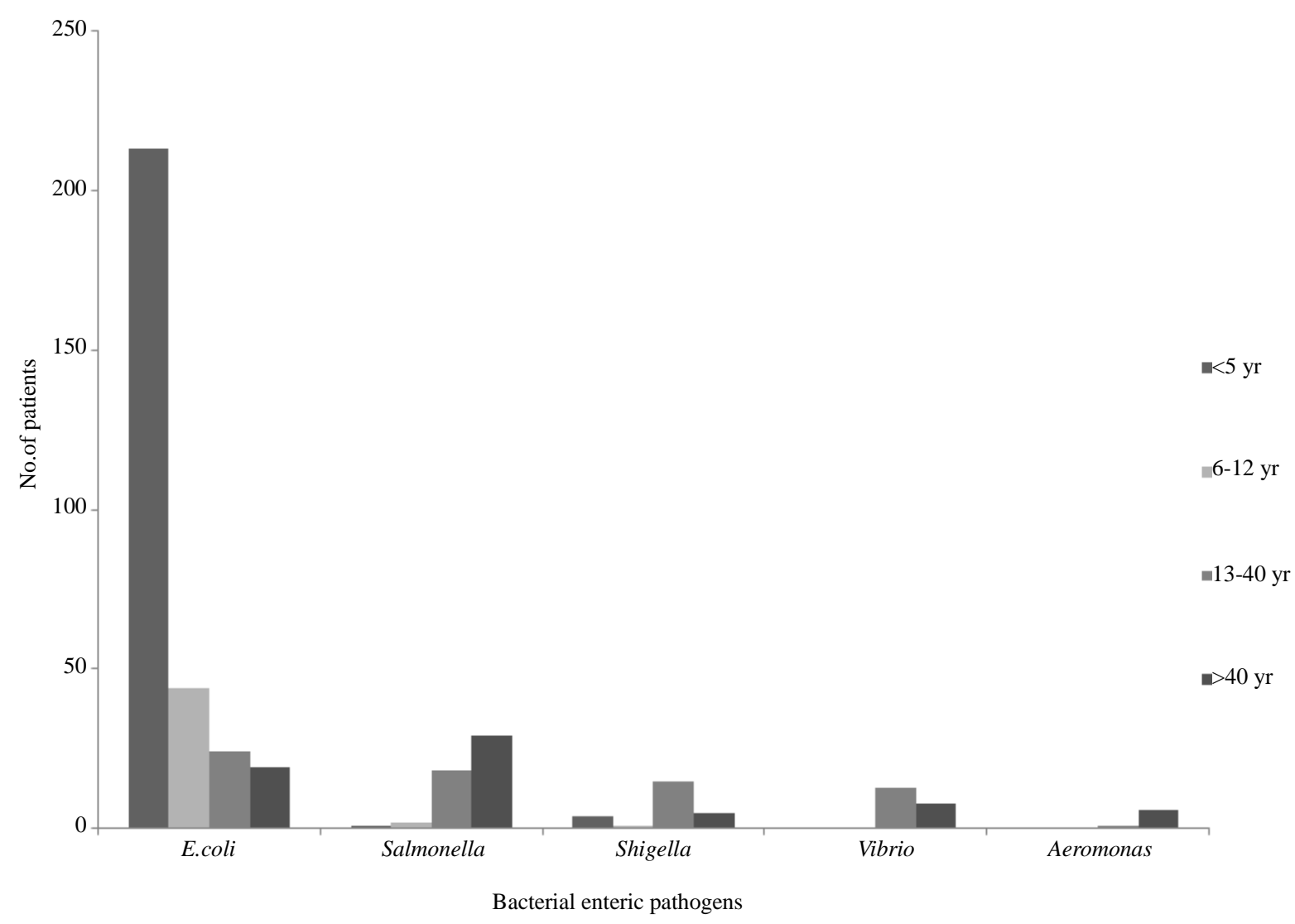

Figure 1. Age wise distribution of bacterial enteric pathogens.

Antibiotic susceptibility pattern: Figure 2 shows the resistance to various antibiotics tested. The year wise analysis of the antibiotic resistance pattern of these bacterial enteric pathogens (data not shown) revealed that DEC showed higher resistance to all the antibiotics tested in 2005 and the resistance was seen to decrease during the successive years of our study period. Shigella species showed a less resistance to all the antibiotics tested from 2005 till 2008. However, resistance was seen to increase from 2009 onwards. High resistance was seen in TET, NAL and CIP as $80 \%, 72 \%$ and $68 \%$ respectively (Figure 2). Salmonella isolates showed less resistance to the antibiotics. However, Non Typhoidal Salmonella isolates showed an increase in resistance to NAL, CIP, SXT and AMP. Vibrio isolates showed resistance to all the antibiotics tested. Aeromonas species showed an increase in resistance to all the antibiotics. It was also interesting to note the fluctuating pattern of resistance to antibiotics each year.

\section{Discussion}

The findings of the present study indicate that both recognized as well as newly recognized agents are important causes of diarrhoea in the developing countries. In this study, we have observed a changing trend in the etiological agents causing diarrhoea. The prevalence of diarrhoea with an identified etiology was $11.2 \%$. Detection of these etiological agents is important for all therapeutic aspects and for implementing appropriate control strategies.

In developing countries DEC are said to be commonly associated with endemic form of diarrhea. The DEC belongs to different categories of pathotypes which are classified based on their clinical features, virulence mechanism and serotypes into EPEC, ETEC, EAEC, EHEC and EIEC. Our earlier study done in May 2001-June 2003 on DEC revealed a higher isolation of EAEC as compared to the other types of DEC [7] [10]. Similar studies done by Banger $\mathrm{R}$ et al. also showed a higher isolation of EAEC among the different types of DEC. The phenotypic and genotypic characteristics were studied to produce an easy method for differentiating strains of EAEC from other strains of DEC [8] [11]. This age related pattern of pathogens is consistent with reports from 


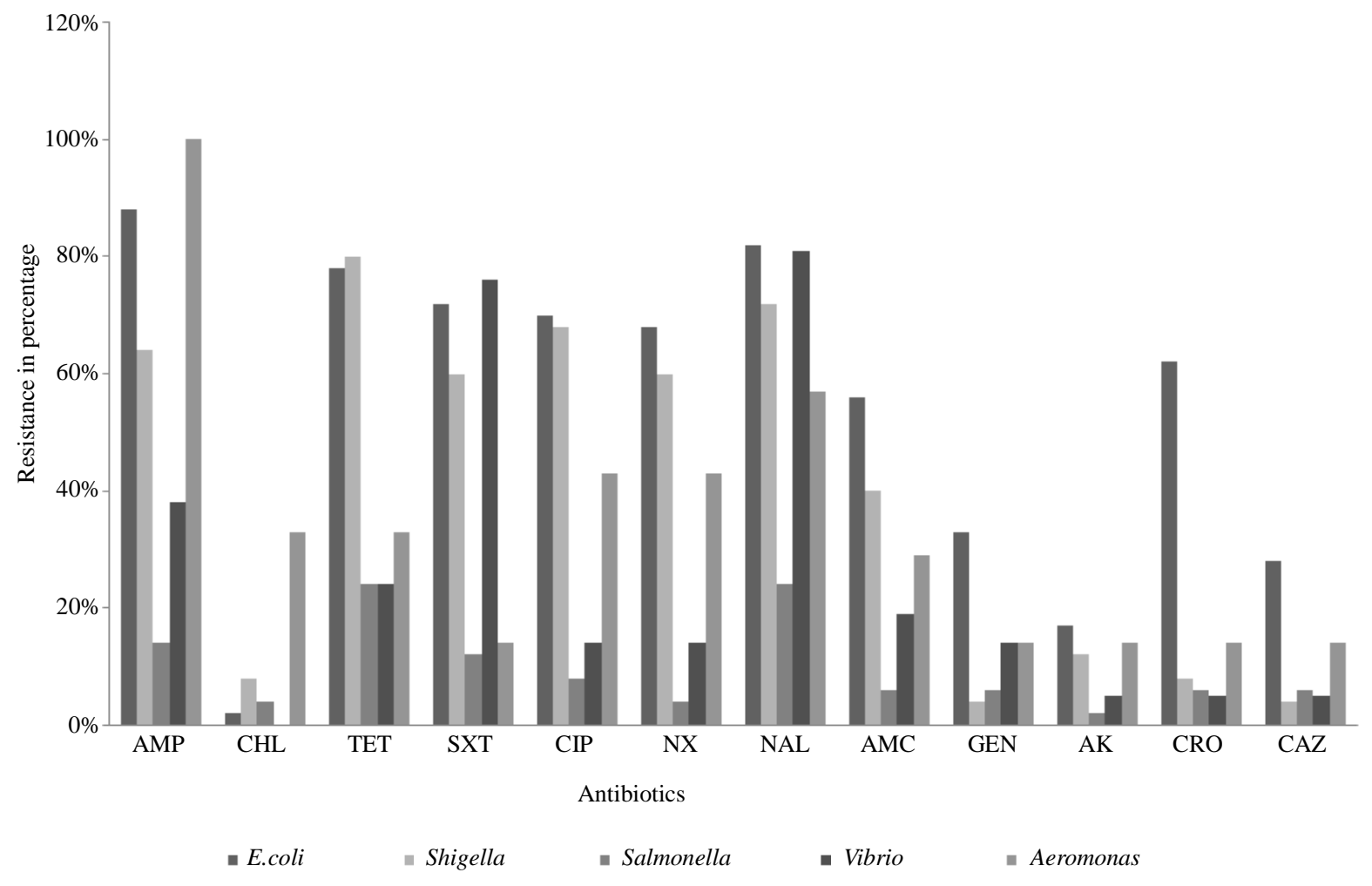

Figure 2. Antibiotic resistance pattern of different bacterial enteric pathogens.

studies conducted in other developing countries [12] [13]. It was also noted that the EAEC strains were mostly multidrug resistant [14].

In agreement with other reports, amongst the non-epidemic diarrheal illness, Shigella and non typhoidal Salmonella gastroenteritis are still the well-recognized etiological agents both in children as well as adults. The prevalence of non-typhoidal Salmonella found in this study (76\%) is higher as compared to our previous observation. The transfer of Salmonella and other zoonotic pathogens to humans via the food supply is an ongoing public concern worldwide. Multi-drug resistant non-typhoidal Salmonella species are widespread all over the world. Study done in north India by Taneja et al. revealed resistance to multiple drugs and an increased resistance to ciprofloxacin [15]. Study done in Mexico by Zaidi M B et al. revealed a high prevalence of Salmonella in retail meat as well as in ill and asymptomatic children. PFGE analysis demonstrated identical DNA banding patterns between them [16].

Shigella infection was significantly associated with older age group (13 - 40 years). S. flexneri and S. sonnei were commonly associated with diarrhoea. $S$. dysentriae was rarely isolated in this study. Our previous study (2000-2006) showed S. flexneri as the predominant serogroup and its resistance to TET, SXT, AMP and NA [17]. Emergence of MDR in Shigella is a global problem. Studies done by Nair et al. showed Shigella sensitive to ceftriaxone [3] contradicting our findings where resistance is seen from 2011 onwards. Reports from north India mention that ESBL producing Shigella strains being isolated and conferring resistance to third generation cephalosporin [18]. Shigellosis in this part of the world is likely to be untreatable disease if this pattern of multiple drug resistance continues. Emergence of MDR strains has been reported due to different genetic factors including transfer of plasmids, integrons and allelic variation in the specific genes [3].

Though cholera is often thought as a major cause of child deaths due to diarrhoea, most cases occur among adults and older children [19]. Cholera was significantly associated with older age group ( $>21$ years). In our observation from 2004-2012, there was a shift in the serotype Inaba to Ogawa and change in the resistance pattern to NA, SXT, AMP, CHL and TET. A large number of cholera epidemics have been associated with multipleantibiotic-resistant (MAR) strains of $V$. cholerae [3] [15] [20]. Improving access to safe drinking water and adequate sanitation, as well as promoting good hygiene are key components in preventing diarrhoea.

The prevalence of Aeromonas species in causing diarrhoea like illness may attribute to the similar genus cha- 
racteristics. Drug resistance observed in isolates of Aeromonas spp. to ampicillin was similar to studies reported from Goa and north India [15].

Rotavirus is the most common cause of severe diarrhea in children worldwide. Though most of the Rota viral infections are mild, 1/3rd of hospitalization worldwide are due to Rotavirus diarrhea. In the present study, Rotavirus diarrhea was seen in children less than 2 years of age. Similar studies also show the isolation of the Rotavirus type A in infants of $>2$ years of age [21] [22]. Unlike, the reports from the mentioned studies, we have isolated Rotavirus as the sole pathogen (8.7\%) as the etiology of diarrhea.

Immunocompromised status provides a suitable environment for the opportunistic infections. Diarrhoea is a common manifestation of HIV infection in both adults and children. In adults, it is usually the consequence of a deteriorating immune system late in the HIV cycle. The present study was compared to our previous laboratory data where a change in the pattern of prevalence of coccidian parasite from Cryptosporidium to Isospora belli in HIV infected individuals was seen. Prevalence of E.histolytica was low in this study and this may be related to the current use of chloroquine under malaria eradication program of Ministry of Health and Family Welfare [3].

In the present study enteric parasites of $5.7 \%$ was isolated, as compared to Candida species comprising $18.5 \%$. Despite the majority of candidiasis cases being attributed to C. albicans, an increasing number of infections caused by non-C. albicans Candida (NCAC) species, specifically Candida glabrata, Candida parapsilosis and Candida tropicalis, have been reported over the past two decades [23]. The association of Candida in causing diarrhoea is not well established and studies show that there may be a possibility of Candidal diarrhoea in selective clinical settings [24] [25].

Our findings show that unusual enteric pathogens being isolated and reported in causing diarrhoea. Vibrio fluvialis has emerged as a rare human pathogen with particular significance in immunocompromised patients, particularly those with AIDS and cancer and in infants. We have reported a case of cholera like illness in a cancer patient [26]. Moraxella osloensis being an environmental strain is rarely known to cause local or invasive infections. A case of diarrhoea in Hemolytic Uremic Syndrome (D + HUS) pediatric child was reported [27]. The rare isolation of Elizabethkingia meningoseptica causing diarrhea in a pediatric child [28] was observed in contrast to the infections associated like meningitis, nosocomial pneumonia and endocarditis. Fasciolopsis, a food borne parasitic zoonosis mainly infects farm pigs. It is most prevalent in school age children. A case of diarrhoea in a retro positive adult with co-infection of Fasciolopsis buski, Taenia spp and Aspergillus spp was reported [29].

Besides the well-known causes of enteric infections and diarrhoeal diseases, parasites and viruses are important etiological agents, which are very often overlooked. These agents have to be identified accurately and promptly so that appropriate treatment modalities could be initiated which would prevent the transmission of infection via feco oral route.

Though much of the work is done by routine phenotypic characterization in the laboratory, molecular diagnosis becomes a necessity for the rapid and accurate diagnosis along with the antimicrobial susceptibility testing. This decreases the overall turnaround time required for routine reporting from 72 hours to less than 48 hours, thus directly reducing the mortality and morbidity rates of diarrheal disease.

The etiology of diarrhea includes both emerging and reemerging pathogens. These agents have to be accurately diagnosed by proper laboratory investigations; otherwise routine treatment of such cases with empirical antibiotics will unnecessarily increase the burden of drug resistance. The change in the antibiotic resistance pattern also cannot be neglected. Empirical drugs once used are now being reported as resistant. The problem of changing resistance patterns in enteric pathogens will remain an ongoing threat for both developed and developing countries. Systematic surveillance and timely reporting of antibiotic resistance patterns among enteric pathogens from different regions of the world should become a high priority. It is important to bear in mind that the principal purpose of monitoring antibiotic resistance trends among enteric pathogens is to provide clinicians with data that can be used to select appropriate treatment regimens.

\section{Conclusion}

Molecular studies have to be considered as a part of diagnosis for prompt and accurate identification along with epidemiological monitoring. An improvement in the hygiene and sanitation is also essential in decreasing the morbidity due to enteric pathogens. Periodic monitoring of drug resistance in enteric pathogens should be carried out in different geographic areas so that an appropriate agent can be chosen for empiric therapy. There is a 
need to educate both the general public and health practitioners that most diarrhoea does not require antibiotics.

\section{Acknowledgements}

We are grateful to Dr. T. Ramamurthy, Deputy Director, Division of Microbiology, National Institute of Cholera and Enteric Diseases, Kolkata, and Dr. Yashwanth Takur, Director, National Salmonella \& E. coli Reference Centre, Central Research Institute, Kasauli for serotyping Salmonella strains.

\section{References}

[1] Ghimire, M. (2008) Acute Diarrhoea and Respiratory Infections: Neglected and Forgotten Health Problems? Communicable Disease Newsletter, 5, 1-2.

[2] Deen, J.L., Von Seidlein, E.L., Sur, D.E., Agtini, M., Lucas, M.E., Lopez, A.L., et al. (2008) The High Burden of Cholera in Children: Comparison of Incidence from Endemic Area in Asia and Africa. PLOS Neglected Tropical Diseases, 2, e173. http://dx.doi.org/10.1371/journal.pntd.0000173

[3] Nair, G.B., Ramamurthy, T., Bhattacharya, M.K., Krishnan, T., Ganguly, S., Rajendran, K., et al. (2010) Emerging Trends in the Etiology of Enteric Pathogens as Evidenced from an Active Surveillance of Hospitalized Diarrhoeal Patients in Kolkata, India. Gut Pathogens, 2, 4. http://dx.doi.org/10.1186/1757-4749-2-4

[4] Das, S., Saha, R. and Singhal, S. (2010) Enteric Pathogens in North Indian Patients with Diarrhoea. Indian Journal of Community Medicine, 32, 1-8.

[5] Cheesebrough, M. (2006) District Laboratory Practices in Tropical Countries. Part 2. 2nd Edition. Cambridge University Press, New York. http://dx.doi.org/10.1017/CBO9780511543470

[6] WHO Manual (2012) http://www.antimicrobialresistance.dk/data/images/protocols/gfn_biochem_final.pdf

[7] Ballal, M. and Ramamurthy, T. (2005) Enteroaggregative Escherichia coli Diarrhea in Manipal. Indian Pediatrics, 42, 722-723.

[8] Bangar, R. and Ballal, M. (2008) Identification of Enteroaggregative Escherichia coli in Infants with Acute Diarrhea Based on Biofilm Production in Manipal, South India. Indian Journal of Medical Sciences, 62, 8-12. http://dx.doi.org/10.4103/0019-5359.38916

[9] Clinical and Laboratory Standards Institute (2013) Performance Standards for Antimicrobial Susceptibility Testing. 15th Informational Supplement, 25, M100-S15.

[10] Ballal, M. and Ramamurthy, T. (2007) Virulence Characteristics and Molecular Epidemiology of Diarrheogenic Escherichia coli (DEC) Associated with Sporadic Cases in a Tertiary Care Hospital in Manipal-Southern India. World Journal of Medical Sciences, 2, 63-64.

[11] Raju, B. and Ballal, M. (2007) Biochemical Identification of Enteroaggregative Escherichia coli among Infants with Acute Diarrhea from Manipal, India. International Journal of Health Sciences, 1, 237-241.

[12] Moyo, S.J., Njolstad, G., Mecky, I.M., Jesse, K., Helge, M., Haima, M., et al. (2011) Age Specific Etiological Agents of Diarrhoea in Hospitalized Children Aged Less than Five Years in Dar es Salaam, Tanzania. BMC Pediatrics, 11, 19. http://dx.doi.org/10.1186/1471-2431-11-19

[13] Guerrant, R.L., Hughes, J.M., Lima, N.L., Crane, J., et al. (1990) Diarrhoea in Developed and Developing Countries: Magnitude, Special Settings and Etiologies. Reviews of Infectious Diseases, 12, 41-50.

http://dx.doi.org/10.1093/clinids/12.Supplement_1.S41

[14] Raju, B. and Ballal, M. (2009) Multidrug Resistant Enteroaggregative Escherichia coli Diarrhoea in Rural Southern Indian Population. Scandinavian Journal of Infectious Diseases, 41, 105-108. http://dx.doi.org/10.1080/00365540802641856

[15] Taneja, N., Mohan, B., Khurana, S., Sharma, M., et al. (2004) Antimicrobial Resistance in Selected Bacterial Enteropathogens in North India. Indian Journal of Medical Research, 120, 39-43.

[16] Zaidi, M.B., McDermott, P.F., Fedorka-Cray, P., Leon, V., Canche, C., Hubert, S.K., Abbott, J., Leon, M., Zhao, S., Headrick, M. and Tollefson, L. (2006) Nontyphoidal Salmonella from Human Clinical Cases, Asymptomatic Children and Raw Retail Meats in Yucatan, Mexico. Clinical Infectious Diseases, 42, 21-28. http://dx.doi.org/10.1086/498508

[17] Mamatha, B., Pusapati, B.R. and Riturparna, C. (2007) Changing Patterns of Antimicrobial Susceptibility of Shigella Serotypes Isolated from Children with Acute Diarrhea in Manipal, South India, a 5 Year Study. Southeast Asian Journal of Tropical Medicine and Public Health, 38, 863-866.

[18] Urvashi, M., Saxena, S. and Dutta, R. (2011) Antimicrobial Resistance Pattern of Shigella Species over Five Years at a Tertiary-Care Teaching Hospital in North India. Journal of Health, Population and Nutrition, 29, 292-295.

http://dx.doi.org/10.3329/jhpn.v29i3.7878 
[19] UNICEF/WHO (2009) Diarrhoea: Why Children Are Still Dying and What Can Be Done. http://www.who.int/publications/2009/9789241598415_eng.pdf

[20] Faruque, S.M., Islam, M.J., Ahmad, Q.S., Biswas, K., Faruque, A.S.G., Nair, G.B., Sack, R.B., Sack, D.A. and Mekalanos, J.J. (2006) An Improved Technique for Isolation of Environmental Vibrio cholera With Epidemic Potential: Monitoring the Emergence of a Multiple-Antibiotic-Resistant Epidemic Strain in Bangladesh. Journal of Infectious Diseases, 193, 1029-1036. http://dx.doi.org/10.1086/500953

[21] Ballal, M. and Shivananda, P.G. (2002) Rotavirus and Enteric Pathogens in Infantile Diarrhoea in Manipal, South India. Indian Journal of Pediatrics, 69, 393-396. http://dx.doi.org/10.1007/BF02722628

[22] Delfina, U., Octavio, A., Gregorio, Y., et al. (2003) Rotavirus Type A and Other Enteric Pathogens in Stool Samples from Children with Acute Diarrhea on the Colombian Northern Coast. International Microbiology, 6, 27-32.

[23] Pfaller, M.A. and Diekema, D.J. (2007) Epidemiology of Invasive Candidiasis: A Persistent Public Health Problem. Clinical Microbiology Reviews, 20, 133-163. http://dx.doi.org/10.1128/CMR.00029-06

[24] Levine, J., Dykoski, R.K. and Janoff, E.N. (1995) Candida-Associated Diarrhea: A Syndrome in Search of Credibility. Clinical Infectious Diseases, 21, 881-886. http://dx.doi.org/10.1093/clinids/21.4.881

[25] Forbes, D., Ee, L., Camer-Pesci, P. and Ward, P.B. (2001) Fecal Candida and Diarrhoea. Archives of Disease in Childhood, 84, 328-331. http://dx.doi.org/10.1136/adc.84.4.328

[26] Ballal, M., Bangar, R., Martena, S., Sarkar, S., Bairy, I., et al. (2010) Vibrio fluvialis from an Immunocompromised Patient in Manipal, South India. Clinical Microbiology Newsletter, 32, 103-104. http://dx.doi.org/10.1016/j.clinmicnews.2010.06.002

[27] Ballal, M. and Martena, S. (2013) First Case Report of Moraxella osloensis Diarrhoea in a Hemolytic Uremic Syndrome/ Acute Renal Failure Child from Rural Coastal India-Manipal, Karnataka. Indian Journal of Pediatrics, 80, $255-257$. ttp://dx.doi.org/10.1007/s12098-011-0672-x

[28] Ballal, M., Chakraborty, R., Mundkur, S., Aroor, S., Balakrishnan, A. and Rajalingam, V. (2013) An Unusual Presentation of Acute Gastroenteritis Caused by Elizabethkingia Meningoseptica in a Child with Sepsis from Rural Karnataka-A Case Report. JIMSA, 26, 114-115.

[29] Ballal, M. and Martena, S. (2012) An Unusual Presentation of Diarrhoea by Fasciolopsis buski, Taenia spp. and Aspergillus spp. In a Retro Positive Patient. A First Case Report from Rural Coastal India-Manipal, Karnataka. Annals of Tropical Medicine and Public Health, 5, 543-545. http://dx.doi.org/10.4103/1755-6783.105160 